Article

\title{
Longitudinal Magneto-Optical Kerr Effect of Nanoporous CoFeB and W/CoFeB/W Thin Films
}

\author{
Weiwei Zhang ${ }^{1,2,3}{ }^{(D}$, Zhanghua Chen ${ }^{1}$, Vladimir I. Belotelov ${ }^{4,5,6}$ a and Yujun Song $1,7, *(\mathbb{D})$
}

1 Research Center of Modern Physics Technology, Beijing Engineering Research Center of Detection and Application for Weak Magnetic Field, School of Mathematics and Physics, University of Science and Technology Beijing, 30 Xueyuan Road, Beijing 100083, China; 13687641416@126.com (W.Z.); chenzhanghua@ustb.edu.cn (Z.C.)

2 School of Mathematics and Physics, Hebei GEO University, 136 East Huai'an Road, Yuhua District, Shijiazhuang 050031, China

3 Shunde Graduate School, University of Science and Technology Beijing, 2 Daliang Zhihui Road, Shunde Distinct, Foshan 528399, China

4 Physics and Technology Institute, Vernadsky Crimean Federal University, Vernadskogo av. 4, 295007 Simferopol, Russia; belotelov@physics.msu.ru

5 NTI Center for Quantum Communications, National University of Science and Technology MISiS, Leninsky Prospekt 4, 119049 Moscow, Russia

6 Photonic and Quantum Technologies School, Lomonosov Moscow State University, Leninskie Gori, 119991 Moscow, Russia

7 Key Laboratory of Pulsed Power Translational Medicine of Zhejiang Province, Hangzhou 310000, China

* Correspondence: songyj@ustb.edu.cn

check for updates

Citation: Zhang, W.; Chen, Z.; Belotelov, V.I.; Song, Y. Longitudinal Magneto-Optical Kerr Effect of Nanoporous $\mathrm{CoFeB}$ and W/CoFeB/W Thin Films. Coatings 2022, 12, 115. https://doi.org/ 10.3390/coatings12020115 Academic Editor: Grzegorz Dercz

Received: 11 December 2021 Accepted: 17 January 2022

Published: 20 January 2022

Publisher's Note: MDPI stays neutral with regard to jurisdictional claims in published maps and institutional affiliations.

Copyright: (C) 2022 by the authors. Licensee MDPI, Basel, Switzerland. This article is an open access article distributed under the terms and conditions of the Creative Commons Attribution (CC BY) license (https:// creativecommons.org/licenses/by/ $4.0 /)$.

\begin{abstract}
Nanoporous $\mathrm{Co}_{40} \mathrm{Fe}_{40} \mathrm{~B}_{20}(\mathrm{CoFeB})$ and sandwich tungsten (W)/CoFeB/W thin films were fabricated via an anodic aluminum oxide (AAO) template-assisted magneto sputtering process. Their thickness-dependent magneto-optical Kerr effect (MOKE) hysteresis loops were investigated for enhanced Kerr rotation. Control of the Kerr null points of the polarized reflected light can be realized via the thicknesses of the $\mathrm{CoFeB}$ layers and $\mathrm{W}$ layers. Simulation of the thickness-dependent phase difference change by the finite element method reveals the existence of the two Kerr null points for $\mathrm{W} / \mathrm{CoFeB} / \mathrm{W}$ thin films, matching the experimental result very well. However, there are two additional Kerr null points for pure CoFeB thin films according to the simulation by comparing with the experimental result (only one). Theoretical analysis indicates that the different Kerr null points between the experimental result and the simulation are mainly due to the enhanced inner magnetization in the ferromagnetic CoFeB layer with the increased thickness, which is usually omitted in the simulation. Clearly, the introduction of non-ferromagnetic W layers can experimentally regulate the Kerr null points of ferromagnetic thin films. Moreover, construction of $\mathrm{W} / \mathrm{CoFeB} / \mathrm{W}$ sandwich thin films can greatly increase the highest magneto-optical susceptibility and the saturated Kerr rotation angle when compared with $\mathrm{CoFeB}$ thin films of the same thickness.
\end{abstract}

Keywords: magneto-optical Kerr effect; magnetization reversal; Kerr null point; nanoporous structure; magnetic thin films; tungsten

\section{Introduction}

Apart from various heterogeneous structures, including distributed periodic systems, lumped systems, irregular and coupled waveguide systems, and controlled magnetic structures, which are available for data processing and storage in the microwave range [1], the magneto-optical Kerr effect (MOKE) describing the linearly polarized light rotation of the reflection and the relative changes in reflectivity [2-4] has been intensively used in optical and magnetic data storage, domain observation [5,6], optical isolators, and fast optical modulation $[7,8]$. Recently, with the acquisition of high sensing performance in small molecules at low concentrations, MOKE has been widely investigated to enhance the 
sensing performance on the gas detection [9,10], liquid detection [11-13], magnetometry, magnetic phase probes [14-16], and magnetic biosensing [17-21] due to its higher figure of merit (FOM) and sensitivity (they are comprehensive parameters for evaluating sensor performance) than conventional optical-mode or magnetic-mode methods. After summarizing the sensing principle in the MOKE spectrum, it is noteworthy that the MOKE-based sensors always exhibit remarkable sensitivity, flexible light phase, polarization modulation, and a large FOM near the position where the MOKE rotation inversion appears [22,23], which is here called the Kerr null point. As the magnetization reversal, finding the Kerr null point shall be of great interest for developing the ultrasensitive technique and the laser-induced magnetization process for potential magneto-electronic and magneto-optical device applications [24-26]. In addition, Kerr null points shall imply lots of information about magnetic anisotropy, exchange bias coupling, and the polarized laser-matter interaction under field since they are related to the fundamentals of magnetization reversal, the interference of reflected light, and the light-matter interaction of magnetic and/or magneto-optical thin films under a magnetic field [26-28]. All-optical magnetization reversal in a $20 \mathrm{~nm}$ thick $\mathrm{Ni}$ film using a circularly polarized light has been demonstrated, with a reversal speed of $260 \mathrm{fs}$, which is much faster than that in other devices, such as magnetic tunneling junctions (MTJs) and giant magnetoresistance (GMR) junctions [29]. The $[\mathrm{Co}(0.4) / \mathrm{Pt}(0.7)]_{n}$ (thickness in $\mathrm{nm}, n \leq 8$ ) multilayer thin films also show magneto-optical magnetization reversal [30]. While many composite iron oxide compounds have excellent magnetic properties [31,32], alloying compounds have several disadvantages. One of the disadvantages is that they are less corrosive to environmental factors, such as temperature, oxygen, and electromagnetic radiation. In this sense, oxides are more stable when used below $1000{ }^{\circ} \mathrm{C}$. However, metal alloys and composites have excellent magneto-optical properties. This means a new method for the magneto-optical control of ferromagnetic magnetization applicable for integrated magneto-optical data [33]. Kerr null points shall promise an alternative favoring the easy manipulation of the magneto-optical control of magnetization.

It is beyond doubt that magneto-optical Kerr rotation reversal is particularly more complex in multilayered ferromagnetic and nonmagnetic systems with large magnetic anisotropy dispersion and an interfacial coupling effect than magnetization reversal, since the phenomena are related to light polarization, light-matter interaction, and interference of the reflected light under external field and inner magnetization of materials [24-28,30,33,34]. Precise regulation of this kind of MOKE rotation reversal thus becomes very important for the study of this complex phenomenon for the optimization of the microstructure and composition of magnetic thin films at nanoscale [24-30,33,34]. Of particular interest is the design and fabrication of special nano-structured thin films, including magnetic layers, for the regulation of MOKE rotation inverse and the related reversal mechanism study. To date, various nanostructures, including periodic rectangular apertures [35], optical Tamm-state structures [36,37], and prism-coupled surface-plasmon-resonance systems [38,39], have been investigated to enhance sensing performance assisted by a variety of fabrication methods, such as electrochemical deposition [40,41] and magnetron sputtering [42].

Apart from such nanostructures, the nanoporous anodic aluminum oxide template (AAO) [43] can realize the control of light propagation and modulate magneto-optical (MO) effects by tailoring the pore diameter, interpore spacing, pore length, and coating composition of multilayered MO media [44-48]. Herein, two types of nanoporous $\mathrm{MO}$ systems, i.e., $\mathrm{CoFeB} / \mathrm{AAO} / \mathrm{Al}$ and $\mathrm{W} / \mathrm{CoFeB} / \mathrm{W} / \mathrm{AAO} / \mathrm{Al}$, were developed to investigate the possibility of controlling the null points of MO Kerr signals to achieve future high-performance sensors by regulating the phase change of the two reflection coefficients (i.e., the original $\mathrm{s}$-composition and p-composition induced by the external magnetic field) and tailoring the film thickness and the interface magnetic and dielectric property. In this work, $\mathrm{CoFeB}$ film has excellent magneto-optical properties and in-plane uniaxial magnetic anisotropy [49], which can be induced by both stress and post-deposition annealing under a magnetic condition, leading to a significant magneto-impedance effect at a low frequency [50]. 
The strategy provides an alternative technique for the fabrication of smart and accurate MOKE sensors for film thickness measurement and a possible new method in the regulation of magnetization reversal via the optimization of the microstructure of multilayered thin films.

\section{Experimental Section}

\subsection{Sample Fabrication and Characterization}

To date, there are various methods for obtaining metallic alloy samples $[40,41]$. In this work, several single ferromagnetic CoFeB films and W (x)/CoFeB (120 nm)/W (x) trilayers were deposited on nanoporous AAO (pore diameter, $90 \mathrm{~nm}$; inter-pore distance, $110 \mathrm{~nm}$; pore length, $300 \mathrm{~nm}$ ) based on an Al substrate (AAO/Al) by magnetron sputtering at room temperature and a base pressure of $4.7 \times 10^{-4} \mathrm{~Pa}$. The CoFeB target was sputtered in a 0.75 mTorr argon gas atmosphere using a radio frequency (RF) sputtering apparatus (Shenyang Pengcheng Vacuum Technology Co., Ltd., Shenyang, China) with a power density of $5.1 \mathrm{~W} / \mathrm{cm}^{2}$. The $\mathrm{W} / \mathrm{CoFeB} / \mathrm{W}$ trilayers were deposited layer-by-layer from bottom to top. The $\mathrm{W}$ target was sputtered in a 15 mTorr Ar gas atmosphere using a direct current (DC) sputtering apparatus with a power density of $2.55 \mathrm{~W} / \mathrm{cm}^{2}$. After the fabrication process, surface, and cross-sectional morphologies and compositional analyses of the $\mathrm{CoFeB} / \mathrm{AAO} / \mathrm{Al}$ and $\mathrm{W} / \mathrm{CoFeB} / \mathrm{W} / \mathrm{AAO} / \mathrm{Al}$ multilayer systems were performed using a field-emission (FE) scanning electron microscope (SEM) (SUPRA 55, Zeiss, Oberkochen, Baden-Württemberg, Germany). The MO effects were investigated using a Kerr measurement instrument (NanoMOKE-3, Durham Magneto Optics Ltd., Durham University Library, Stockton Road, Durham Dh1 3ly, United Kingdom) working in a longitudinal geometry (L-MOKE). As shown in Figure 1, the external magnetic field was parallel to + incident light. In our system, longitudinal MO hysteresis loops for various systems were recorded at a $660 \mathrm{~nm}$ wavelength at a $45^{\circ}$ angle of incidence in s-polarization for different magnetic-field strengths.

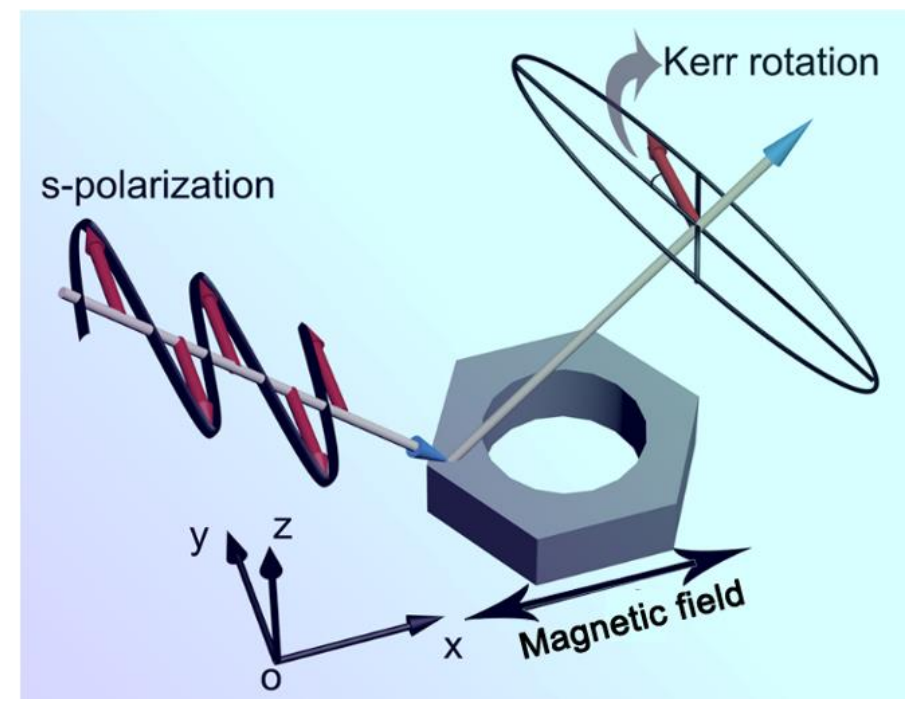

Figure 1. Longitudinal magneto-optical measurement configuration.

\subsection{Simulation}

The MO properties of the $\mathrm{CoFeB} / \mathrm{AAO} / \mathrm{Al}$ and $\mathrm{W} / \mathrm{CoFeB} / \mathrm{W} / \mathrm{AAO} / \mathrm{Al}$ multilayer systems were simulated by the finite element method using COMSOL Multiphysics (version: 5.6) [51]. An equation deduced using the transfer matrix method from a semi-infinite layer was employed to approximately calculate the Kerr signal [52]. For the incident beam 
in the s-polarization, the Kerr rotation $\theta$ and the ellipticity $\varphi$ were simulated as functions of the reflection coefficients $r_{i j}(i, j=s, p)$ as follows:

$$
\begin{aligned}
& \theta=\frac{1}{2}\left(\tan ^{-1}\left(\frac{2 \operatorname{Re}\left(\frac{r_{p s}}{r_{s s}}\right)}{\left(1-\left|\frac{r_{p s}}{r_{s s}}\right|^{2}\right)}\right)\right) \\
& \varphi=\frac{1}{2}\left(\sin ^{-1}\left(\frac{2 \operatorname{Im}\left(\frac{r_{p s}}{r_{s s}}\right)}{\left(1+\left|\frac{r_{p s}}{r_{s s}}\right|^{2}\right)}\right)\right)
\end{aligned}
$$

where the modulus $\left|\frac{r_{p s}}{r_{s s}}\right|$, the real part $\operatorname{Re}\left(\frac{r_{p s}}{r_{s s}}\right)$, and the imaginary part $\operatorname{Im}\left(\frac{r_{p s}}{r_{s s}}\right)$ are considered close to zero. Then, the Kerr signal $\Psi$ can be expressed as follows:

$$
\Psi=\frac{r_{p s}}{r_{s S}}=\theta+\varphi i=\rho e^{\delta i}=\rho \cos (\delta)+\rho \sin (\delta) i
$$

where, $\rho$ is the modulus of the reflection coefficient ratio; $\delta$ is the phase difference of the two components of the reflection light, caused by the interaction of the light with the thin films in which the horizontal component travels at a speed different from the vertical component. From Equation (3), the sign of the Kerr rotation can be controlled by the phase difference $\delta$ that is a function of the film thickness $(t)$, effective refraction index $\left(n_{\text {eff }}\right)$, and MO constant $(Q)$ of $\mathrm{CoFeB}$ film. A simplified computational model is shown in Figure 2. For s-polarized incident light with a wavelength of $660 \mathrm{~nm}$, the simulation needs to account for the interplay of several parameters: (a) the permittivity tensor or complex index of the refraction of every layer, (b) the $\mathrm{MO}$ constant of the $\mathrm{CoFeB}$ layer, and (c) film thicknesses.

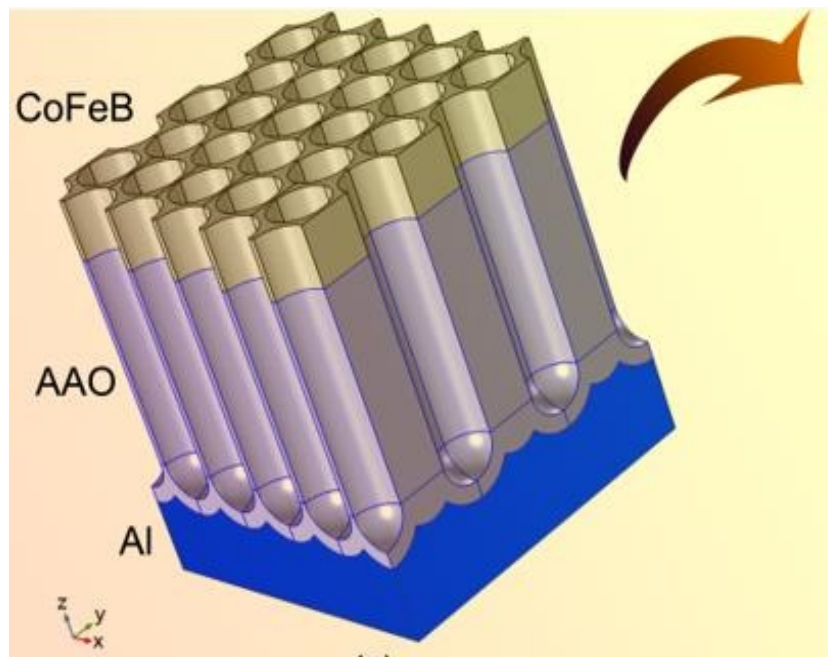

(a)

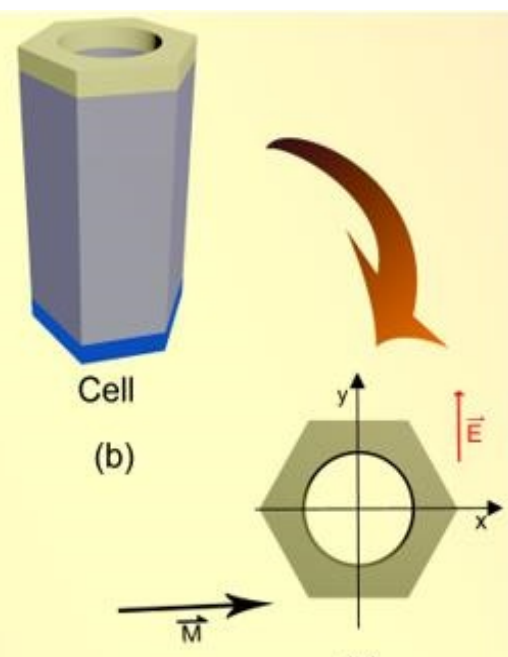

(c)

Figure 2. (a) Hexagonal honeycomb structure covered with a CoFeB film. (b) The periodic cell used to calculate the magneto-optical Kerr signal. (c) A schematic illustration of the electric field $\overrightarrow{\mathrm{E}}$, external magnetic field $\overrightarrow{\mathrm{M}}$, and the sample surface.

For the isotropic $\mathrm{CoFeB}$, the purely optical components can be expressed using $\varepsilon_{x x} \approx \varepsilon_{y y} \approx \varepsilon_{z z} \approx \varepsilon=N^{2}$, where $N$ is the complex refractive index of the magnetic material. All the remaining components equal zero when considering L-MOKE in s-configuration for 
the $\mathrm{CoFeB}$ nanoporous thin film. Thus, the permittivity tensor of CoFeB can be expressed using Equation (4) with an anti-symmetric tensor as follows:

$$
\varepsilon^{L}=\left(\begin{array}{ccc}
\varepsilon & 0 & 0 \\
0 & \varepsilon & -i \varepsilon Q \\
0 & i \varepsilon Q & \varepsilon
\end{array}\right)
$$

Here, $Q$ is activated by magnetization along the $x$-axis, and the appearance of the Kerr rotation originates from off-diagonal terms. The MO coupling constant $Q=1.3 \times 10^{-2}+$ $9.9 \times 10^{-5} i$ of the $\mathrm{CoFeB}$ film can be obtained from a magnetic home-made ellipsometric measurement.

For the theoretical simulations, the diagonal terms of the permittivity tensors for $\mathrm{CoFeB}$ and $\mathrm{W}$ were determined using the equation $N^{2}=(n+i k)^{2}$ (where $n$ is the refractive index and $k$ is the extinction coefficient) by employing the spectral dependence of the complex refractive index of the films obtained from the ellipsometry measurement (Horiba UVISEL, Horiba Scientific, Paris, France). Additionally, when elliptically polarized light is reflected through the film, its polarization state is changed to another elliptically polarized light. The thickness value is finally obtained by obtaining the azimuth angle of the starter and the azimuth angle of the detector, using the fact that the film thickness exists as a function of these two parameters. The wavelength dependent $n$ and $k$ of CoFeB layers and $\mathrm{W}$ layers are plotted in Figure 3. At $660 \mathrm{~nm}$, the values of $n$ and $k$ for CoFeB films are 3.66 and 1.16, respectively, and those for $W$ films are 3.58 and 1.30, respectively. The relative permittivity of $\mathrm{Al}_{2} \mathrm{O}_{3}$ is 3.11 and $\mathrm{Al}$ used in the simulation is obtained from Ref [53].
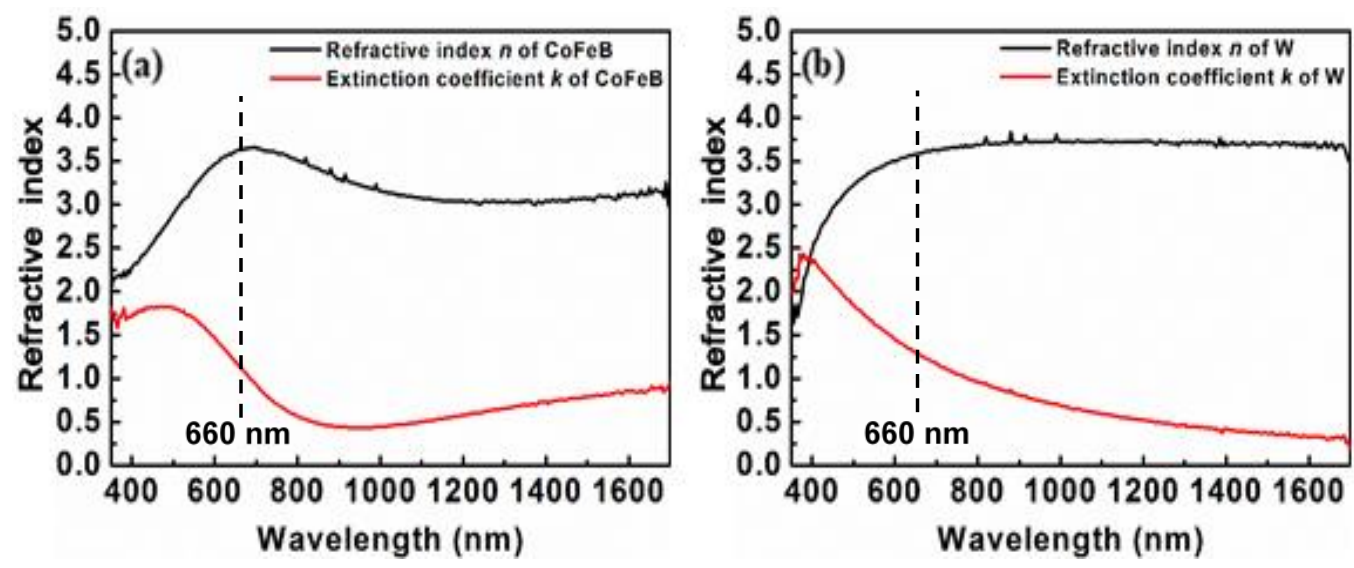

Figure 3. Wavelength-dependent refractive index $n$ and extinction coefficient $k$ of (a) CoFeB layers and (b) W layers.

\section{Results and Discussion \\ CoFeB Thickness Dependent MOKE of Nanoporous CoFeB/AAO/Al Thin Films}

Nanoporous CoFeB/AAO/Al thin films with CoFeB layers with thicknesses from 12 to $372 \mathrm{~nm}$ were fabricated by a magneto-sputtering process. Figure 4a shows one top-viewed image of the pure AAO substrate by SEM (SUPRA 55, Zeiss, Oberkochen, Baden-Württemberg, Germany), revealing the nearly hexagonal arranged nanoporous surface morphology with a pore diameter of $90 \mathrm{~nm}$ and an interpore spacing of $110 \mathrm{~nm}$. The cross-section of the pure AAO substrate reveals uniform long straight holes in the AAO substrate (Figure $4 \mathrm{~b}$ ). Figure $4 \mathrm{c}$, d show the top surface image and the cross-section image of one representative $\mathrm{CoFeB} / \mathrm{AAO}$ thin film with a CoFeB thickness of $51 \mathrm{~nm}$. These images revealed slightly reduced surface nanopore diameters of about $80 \mathrm{~nm}$ and petal-like arrayed nanopore structures that were connected by equilateral triangles with the side length equal to the inter-pore spacing of $110 \mathrm{~nm}$. 

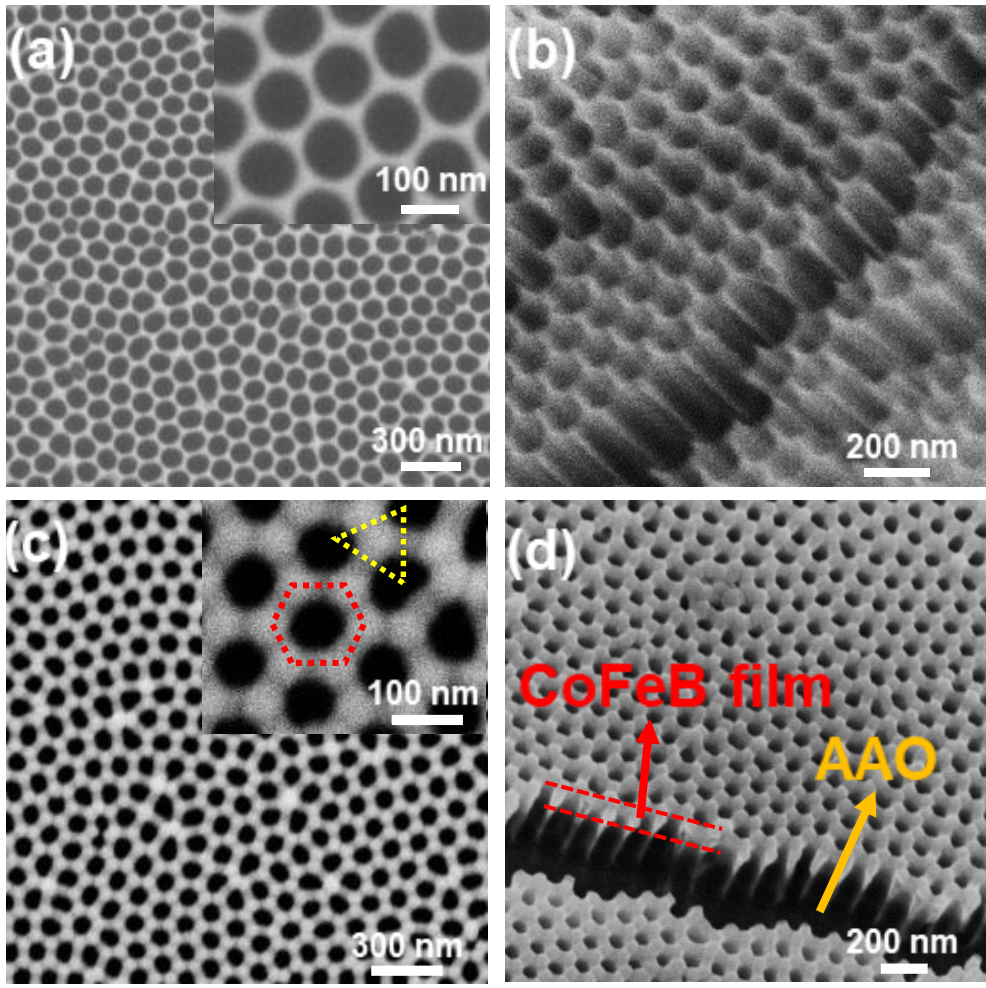

Figure 4. (a) Wide surface and (b) cross section SEM images of a porous AAO film (pore diameter, $90 \mathrm{~nm}$; inter-pore spacing, $110 \mathrm{~nm}$ ) without any coating. (c) Surface SEM image of a $51 \mathrm{~nm}$ thick $\mathrm{CoFeB}$ film fabricated on the AAO substrate of $90 \mathrm{~nm}$ pore diameter. (d) Cross section SEM image of thin films coated with $51 \mathrm{~nm} \mathrm{CoFeB}$ layer on the AAO substrate of $90 \mathrm{~nm}$ pore diameter.

The ferromagnetic layer thickness-dependent MOKE of nanoporous CoFeB/AAO/Al thin films was investigated via the s-polarized longitudinal-MOKE (L-MOKE) hysteresis loops, which are summarized in Figure 5, with the CoFeB layer thickness increasing from 12 to $372 \mathrm{~nm}$. Determining the thickness of thin films on AAO templates is often a challenge. Traditionally, however, researchers have typically deposited thin films on AAO and smooth substrates (e.g., glass and wafer) using the same deposition time. The film thickness on the smooth substrate was then characterized using an optical ellipsometer or atomic force microscope. In our experiments, we used this method to characterize the film thickness on AAO templates. It was surprisingly found that there was one inversion of the Kerr hysteresis loops appearing from 51 to $66 \mathrm{~nm}$, and then no further inversion was observed as the CoFeB thickness was continuously increased up to $372 \mathrm{~nm}$. For the precise critical thickness confirmation of the inversed Kerr hysteresis loop, three more Kerr hysteresis loops with thicknesses of 55, 58, and $61 \mathrm{~nm}$ were further measured. As shown in Figure 6, $\mathrm{CoFeB} / \mathrm{AAO}$ thin films showed a critical thickness between 51 and $55 \mathrm{~nm}$, during which a relative inversion of the Kerr hysteresis loops occurred, suggesting that the MOKE rotation angle can be reduced to zero, so called the Kerr null point. This null point indicates an abrupt MOKE polarization change and/or a magnetization change of thin films as CoFeB thickness is increased from 51 to $55 \mathrm{~nm}$, which may be used for ultrasensitive ellipsometers for the thickness or the related magnetic/optical signal measurement. The estimated $\mathrm{CoFeB}$ thickness for the Kerr null point of $\mathrm{CoFeB}$ thin films is about $51.8 \mathrm{~nm}$ according to the thickness-dependent Kerr rotation angle. 

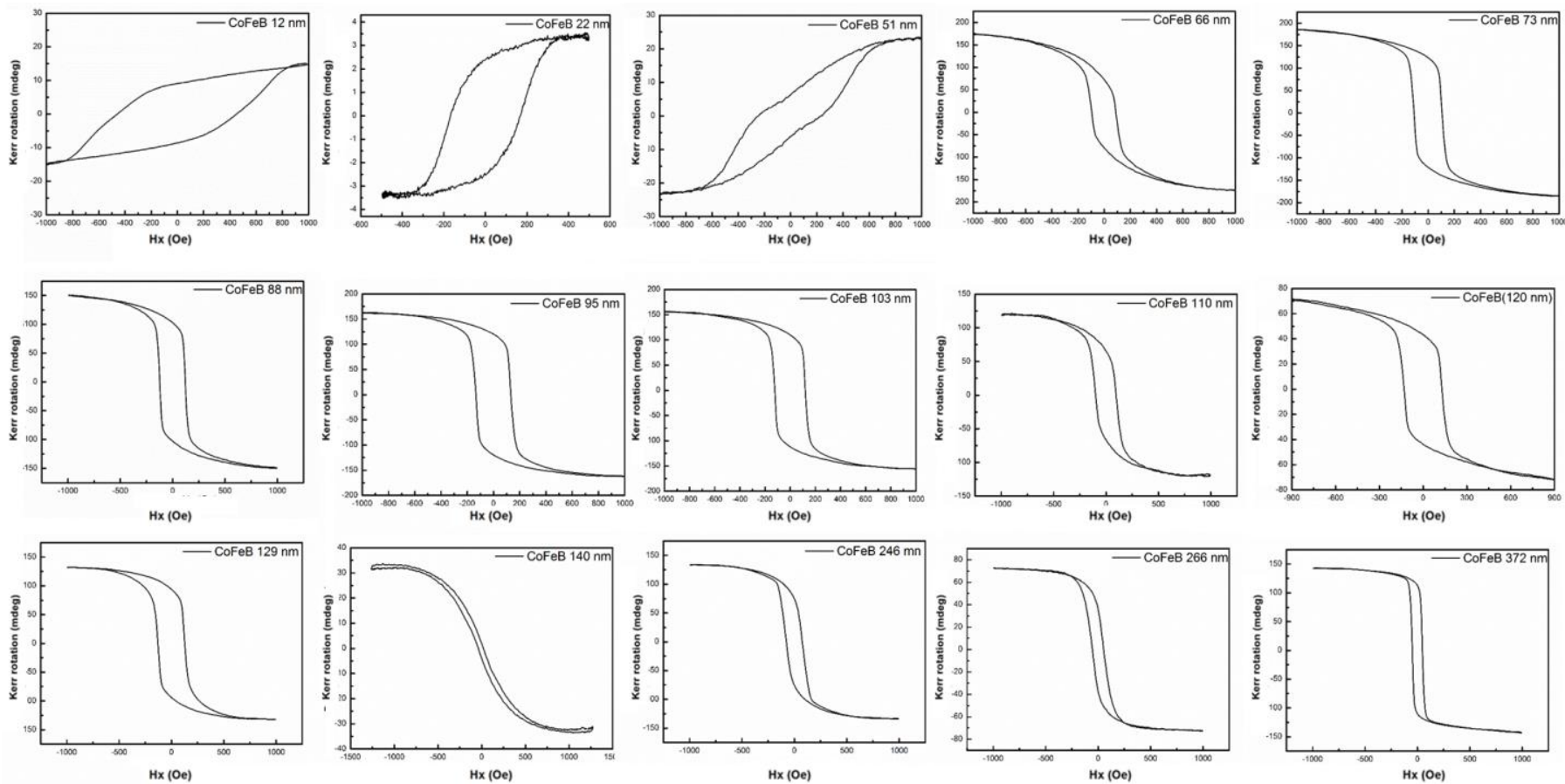

Figure 5. s-polarization L-MOKE hysteresis loops for $\mathrm{CoFeB} / \mathrm{AAO}$ thin films with $\mathrm{CoFeB}$ thicknesses from 12 to $372 \mathrm{~nm}$ (the CoFeB thickness of each film sample is labeled in the upper right of each subfigure), excited at a laser wavelength of $660 \mathrm{~nm}$ and an inducing angle of $45^{\circ}$.
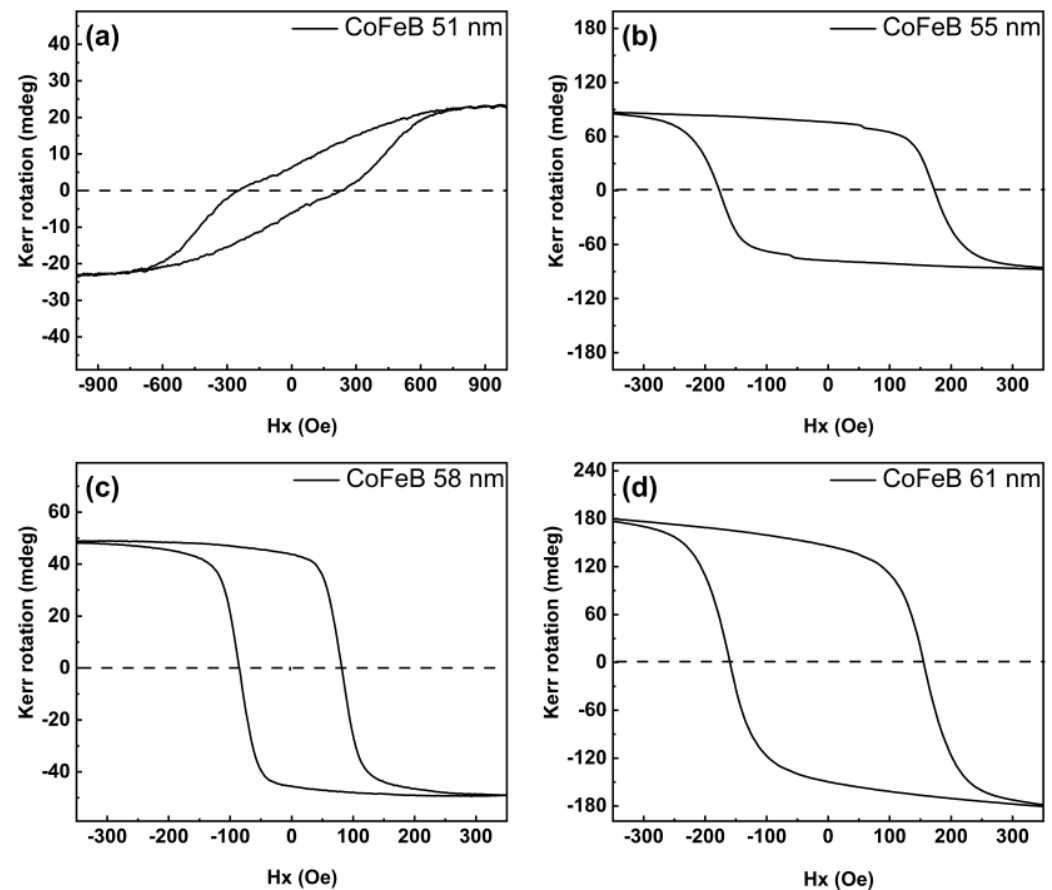

Figure 6. s-polarization L-MOKE hysteresis loops of $\mathrm{CoFeB} / \mathrm{AAO}$ thin films with CoFeB layer thicknesses of (a) 51, (b) 55, (c) 58, and (d) $61 \mathrm{~nm}$, excited at a laser with wavelength of $660 \mathrm{~nm}$ and an inducing angle of $45^{\circ}$.

Although there is no further MOKE hysteresis inverse with the further increase of CoFeB thickness of these nanoporous thin films, their Kerr rotation angle and magnetic susceptibility can be further regulated by the CoFeB thickness. Table 1 gives the highest Kerr susceptibility $(\chi$, mdeg/Oe, the maximum value of slope along the hysteresis cycle, 
from the peaks of the differential curves of the Kerr rotation hysteresis loops), the saturated Kerr rotation angles $\left(\theta_{S}\right.$, mdegree, or mdeg) at the positive saturation magnetic field corresponding to the positive hysteresis loop (e.g., the case with the CoFeB thickness of 51 $\mathrm{nm})$ ), and the mean coercivities ( $\mathrm{Hc}$, the mean value of the left field $(\mathrm{Hc}, \mathrm{L})$ and the right field ( $\mathrm{Hc}, \mathrm{R})$ as the Kerr rotation angles become zero), with the CoFeB thickness increase. The highest saturated Kerr rotation of 179.9 mdeg can be obtained as the CoFeB thickness is increased to $61 \mathrm{~nm}$. The highest magnetic susceptibility of $6.61 \mathrm{mdeg} / \mathrm{Oe}$ can be obtained as the $\mathrm{CoFeB}$ thickness is increased up to $372 \mathrm{~nm}$ and the corresponding coercivity (Hc) of this thin film can be as small as 48.5 Oe, but the saturated Kerr rotation angle is only 131.1 mdeg.

Table 1. CoFeB thickness ( $\mathrm{t}, \mathrm{nm}$ ) depending on the highest MOKE susceptibility ( $\chi$, mdeg/Oe), coercivity $(\mathrm{Hc}, \mathrm{Oe})$, and MOKE saturation rotation angle $\left(\theta_{\mathrm{S}}, \mathrm{mdeg}\right)$ of the $\mathrm{CoFeB}$ thin films on AAO substrates.

\begin{tabular}{ccccccccccc}
\hline $\mathbf{t}$ & $\mathbf{1 2}$ & $\mathbf{2 2}$ & $\mathbf{5 1}$ & $\mathbf{5 5}$ & $\mathbf{5 8}$ & $\mathbf{6 1}$ & $\mathbf{6 6}$ & $\mathbf{7 3}$ & $\mathbf{8 8}$ \\
\hline$\chi$ & 0.04 & 0.05 & 0.09 & -1.96 & -1.61 & -3.61 & -2.36 & -4.56 & -4.34 \\
\hline $\mathrm{Hc}$ & 460.0 & 168.2 & 242.9 & 175.0 & 83.2 & 157.3 & 95.1 & 107.6 & 123.2 \\
\hline$\theta_{\mathrm{S}}$ & 14.5 & 3.3 & 22.6 & -85.0 & -48.6 & -179.9 & -144.9 & -155.0 & -124.7 \\
\hline $\mathrm{t}$ & 95 & 103 & 110 & 120 & 129 & 140 & 246 & 266 & 372 \\
\hline$\chi$ & -4.26 & -4.68 & -1.69 & -1.21 & -2.69 & -0.11 & -1.58 & -0.81 & -6.61 \\
\hline $\mathrm{Hc}$ & 134.1 & 143.2 & 98.3 & 130.5 & 127.8 & 27.9 & 76.6 & 53.2 & 48.5 \\
\hline$\theta_{\mathrm{S}}$ & -145.0 & -138.2 & -106.8 & -70.0 & -130.9 & -32.4 & -125.2 & -70.4 & -131.1 \\
\hline
\end{tabular}

To further seek the Kerr null points for expanded sensing range, metallic $W$ layers were introduced to construct the $\mathrm{W} / \mathrm{CoFeB} / \mathrm{W} / \mathrm{AAO}$ nanoporous thin films by consideration of the high spin-orbit coupling and magnetic pining effect of $W$ to the ferromagnetic layer. A series of $\mathrm{W} / \mathrm{CoFeB} / \mathrm{W} / \mathrm{AAO}$ multilayered nanoporous thin films with the $\mathrm{W}$ thickness ranging from 4 to $126 \mathrm{~nm}$ and a fixed $120 \mathrm{~nm}$ thick $\mathrm{CoFeB}$ middle layer were fabricated by a magnetron-sputtering process. Figure 7a shows a representative surface SEM image of the $\mathrm{W} / \mathrm{CoFeB}(120 \mathrm{~nm}) / \mathrm{W} / \mathrm{AAO}$ thin film with each W layer $129 \mathrm{~nm}$ thick. Clearly, the surface morphology (Figure 7a) showed the reduced pore size of about $10 \pm 2 \mathrm{~nm}$ and a petal-like structure formed by nearly discrete equilateral triangles with side lengths equal to the inter-pore spacing after deposition with two W layers (totally $258 \mathrm{~nm}$ ) and one middle $\mathrm{CoFeB}$ layer of $120 \mathrm{~nm}$ thick. Compared with the pure $90 \mathrm{~nm}$ AAO porous film (Figure $4 \mathrm{~b}$ ) and the $\mathrm{CoFeB} / \mathrm{AAO}$ thin films with $51 \mathrm{~nm}$ thick $\mathrm{CoFeB}$ (Figure 4d), the cross-section SEM image of the $\mathrm{W} / \mathrm{CoFeB} / \mathrm{W} / \mathrm{AAO}$ thin film also indicated the shrunk pore size and the formation of three distinct layers (Figure $7 \mathrm{~b}$ ). A combination of element analysis of the thin films by the energy-dispersive X-ray spectrum (EDS: Figure 7c) and the above SEM images reveals the successful fabrication of $\mathrm{W} / \mathrm{CoFeB} / \mathrm{W} / \mathrm{AAO}$ sandwich nanoporous thin films on AAO substrates. The sandwich thin film has a $\mathrm{Fe} / \mathrm{Co} / \mathrm{W}$ atomic ratio of 3.46/3.52/5 and the $\mathrm{Co} / \mathrm{Fe}$ ratio matches the target composition $\left(\mathrm{Co}_{40} \mathrm{Fe}_{40} \mathrm{~B}_{20}\right)$ very well.

The $\mathrm{W}$ thickness-dependent s-polarization L-MOKE of these $\mathrm{W} / \mathrm{CoFeB}(120 \mathrm{~nm}) / \mathrm{W} / \mathrm{AAO}$ nanoporous thin films were studied. As shown in Figure 8a, the Kerr rotation angle first decreased as the $\mathrm{W}$ thickness increased from 4 to $32 \mathrm{~nm}$. This is evident for sample $\mathrm{W}$ $(32 \mathrm{~nm}) / \mathrm{CoFeB} / \mathrm{W}(32 \mathrm{~nm})$, where the line shape of MOKE hysteresis suggests the presence of two different contributions of opposite signs. In some cases, when two magneto-optical contributions are present, they can be isolated, which has been performed in iron oxide nanoparticles using magnetic circular dichroism, allowing discrimination between magnetite and maghemite [16]. Subsequently, the Kerr rotation angle is increased (Figure $8 b$ ) as the W thickness is increased from 42 to $74 \mathrm{~nm}$. Thus, the Kerr rotation hysteresis loops change their direction as the $W$ thickness increases from 32 to $42 \mathrm{~nm}$, resulting in the first Kerr null point at the $\mathrm{W}$ thickness between 32 and $42 \mathrm{~nm}$. When the $\mathrm{W}$ thickness was continuously increased 
to more than $74 \mathrm{~nm}$, the rotation angle was reduced again, leading to a second Kerr rotation hysteresis loop inverse as the $W$ thickness was increased from 95 to $116 \mathrm{~nm}$ (Figure 8b,c). The second Kerr rotation hysteresis loop inverse leads to a second Kerr null point with the $\mathrm{W}$ thickness between 95 and $116 \mathrm{~nm}$. To unambiguously demonstrate these results, the W thickness-dependent saturated Kerr rotation angles for a positive saturation magnetic field are plotted in Figure 8d, demonstrating two Kerr null points with the estimated critical W thicknesses of $37 \mathrm{~nm}$ and $113 \mathrm{~nm}$, respectively.

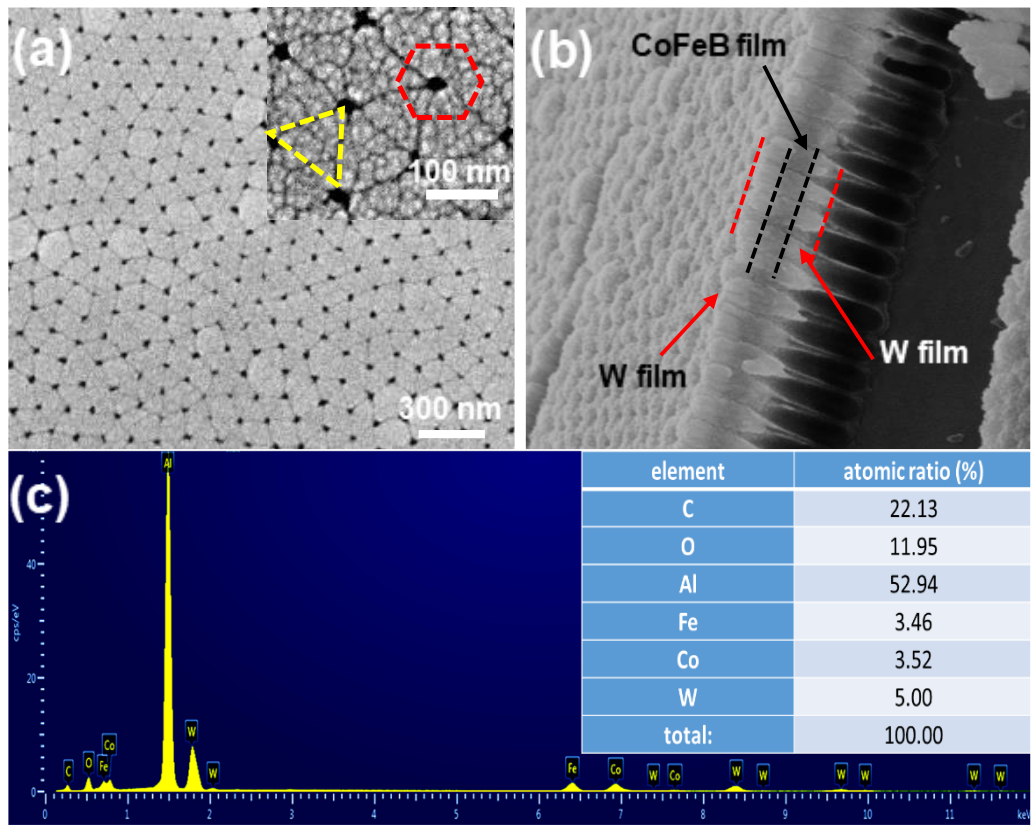

Figure 7. (a) Surface SEM image, (b) cross section SEM image, and (c) surface EDS spectrum of one of W/CoFeB $(120 \mathrm{~nm}) / \mathrm{W}$ multilayer thin films with a W thickness of $129 \mathrm{~nm}$ on the AAO substrate of $90 \mathrm{~nm}$ pore diameter and inter-spacing of $110 \mathrm{~nm}$.
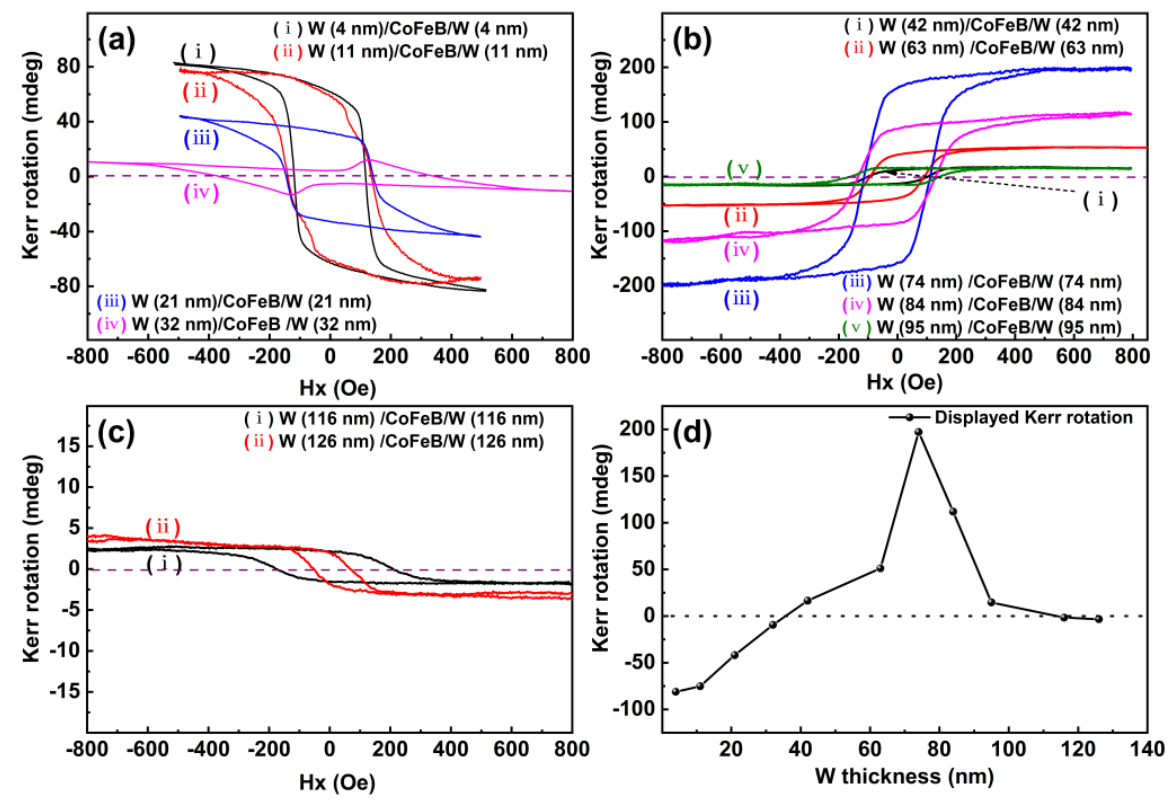

Figure 8. Magneto-optical hysteresis loops versus magnetic-field strength of AAO patterned with multilayered W/CoFeB/W structures. The $W$ thickness is (a) $4 \sim 32 \mathrm{~nm}$, (b) $42 \sim 95 \mathrm{~nm}$, and (c) $116 \sim 126 \mathrm{~nm}$. (d) The $\mathrm{W}$ thickness-dependent saturated Kerr rotation angle at the positive saturation magnetic field corresponding to the positive hysteresis loop (e.g., the case with the $\mathrm{W}$ thickness of $74 \mathrm{~nm}$ ). 
Table 2 gives the highest Kerr susceptibility $(\chi)$, the saturated Kerr rotation angles $\left(\theta_{\mathrm{S}}\right)$ and the mean coercivity $(\mathrm{Hc})$ at different $\mathrm{W}$ thicknesses. The highest $\chi$ for $\mathrm{W} / \mathrm{CoFeB}$ $(120 \mathrm{~nm}) / \mathrm{W}$ nanoporous thin films can be increased from $1.21 \mathrm{mdeg} / \mathrm{Oe}$ to $3.04 \mathrm{mdeg} / \mathrm{Oe}$ as $4 \mathrm{~nm}$ thick $\mathrm{W}$ layers are introduced on the top and bottom of the pure CoFeB nanoporous film. Its coercivity $(\mathrm{Hc})$ can be reduced from $130.5 \mathrm{Oe}$ of the pure CoFeB films to $116.5 \mathrm{Oe}$, correspondingly. $\mathrm{W} / \mathrm{CoFeB} / \mathrm{W}$ nanoporous thin films exhibit the highest Kerr rotation angle of 198.6 mdeg as the $\mathrm{W}$ thickness is increased to $74 \mathrm{~nm}$, higher than that of the pure $\mathrm{CoFeB}$ thin films with the same CoFeB thickness $(70 \mathrm{mdeg})$, which is still higher than the highest Kerr rotation angle of the pure CoFeB thin films with the CoFeB thickness of $61 \mathrm{~nm}$ (179.9 mdeg).

Table 2. The highest susceptivity $(\chi, \mathrm{mdeg} / \mathrm{Oe})$, the coercivity $(\mathrm{Hc}, \mathrm{Oe})$ and the saturated rotation angle $\left(\theta_{S}, \mathrm{mdeg}\right)$ of the $\mathrm{W} / \mathrm{CoFeB}(120 \mathrm{~nm}) / \mathrm{W}$ thin films at different $\mathrm{W}$ thicknesses $(\mathrm{t}, \mathrm{nm})$.

\begin{tabular}{cccccccccccc}
\hline $\mathbf{t}$ & $\mathbf{4}$ & $\mathbf{1 1}$ & $\mathbf{2 1}$ & $\mathbf{3 2}$ & $\mathbf{4 2}$ & $\mathbf{6 3}$ & $\mathbf{7 4}$ & $\mathbf{8 4}$ & $\mathbf{9 5}$ & $\mathbf{1 1 6}$ & $\mathbf{1 2 6}$ \\
\hline$\chi$ & -3.04 & -1.26 & -1.02 & 0.142 & 0.311 & 0.813 & 2.98 & 1.40 & 0.33 & -0.03 & -0.06 \\
\hline $\mathrm{H}_{\mathrm{C}}$ & 116.5 & 142.5 & 145.5 & 354.9 & 101.1 & 153.0 & 111.8 & 131.9 & 151.8 & 194.3 & 59.1 \\
\hline$\theta_{\mathrm{S}}$ & -85.4 & -75.2 & -42.6 & 9.6 & 16.9 & 16.1 & 198.6 & 109.0 & 15.5 & -2.0 & -2.9 \\
\hline
\end{tabular}

Understanding these MOKE features (e.g., layer thickness and composition dependent Kerr inversion and angle rotation) of multilayered ferromagnetic nanoporous films shall pave a new approach for fabricating magnetic nanostructure devices for ultrasensitive MOKE sensing devices. Theoretically, this MOKE hysteresis loop inversion (or experimentally, the susceptibility of Kerr rotation angle changes its direction) can be attributed to an abrupt shift in the phase difference caused by the reflection coefficient change of $r_{p s}$ and $r_{s s}$ (see Equation (3)) and/or by the in plane magnetization direction change of the thin films since $\varepsilon Q$ in Equation (4) is equal to the gyration vector ( $g$, as shown in Equation (5)) [54].

$$
\varepsilon Q=g=\varepsilon_{0} \chi^{(\mathrm{m})} \mathrm{H}
$$

The off-diagonal element $(g)$ represents the magnetically-induced part and is directly proportional to an internal magnetization $(M)$ and the incident wavelength $(\lambda)$, as shown in Equation (6) [54].

$$
g=g(M, \lambda)
$$

Introduction of the non-magnetic double tungsten layers helps to modifies MOKE hysteresis loops of the thin films by solely studying the interference among the light reflected from air- $\mathrm{W} / \mathrm{CoFeB} / \mathrm{W}$ interfaces and $\mathrm{AAO} / \mathrm{Al}$ interfaces regardless of the effect from the internal magnetization of the thin films. This is confirmed by simulations of the cosine values as a function of the $\mathrm{W}$ thickness-dependent $\cos (\delta)$ in the $\mathrm{W} / \mathrm{CoFeB}(120 \mathrm{~nm}) / \mathrm{W} / \mathrm{AAO}$ thin films and the $\mathrm{CoFeB}$ thickness-dependent $\cos (\delta)$ in the $\mathrm{CoFeB} / \mathrm{AAO}$ thin films. Figure $9 \mathrm{a}$ shows that the $\cos (\delta)$ spectra exhibited two null points as the $\mathrm{W}$ thickness was increased from 4 to $140 \mathrm{~nm}$, which occurred at the $W$ thickness of $41 \mathrm{~nm}$ and $86 \mathrm{~nm}$, respectively. The deviation from the experiment values ( 37 and $113 \mathrm{~nm}$ ) is mainly due to neglect of the inplane magnetization of the $\mathrm{CoFeB}$ layer and the pinning effect of the $\mathrm{W}$ layer on the surface magnetization of the CoFeB layer, and partially due to some variation in the $\mathrm{W}$ thickness measurement. The simulation result on the $\cos (\delta)$ spectra for $\mathrm{CoFeB} / \mathrm{AAO}$ nanoporous thin films (Figure 9b) reveals three null points as CoFeB thickness is increased from 12 to $372 \mathrm{~nm}$, which are 24, 128, and $201 \mathrm{~nm}$, which is different from the experimental result. It will not be surprising as you consider the fact that the simulation only considers the light reflected from the air- $\mathrm{CoFeB} / \mathrm{AAO} / \mathrm{Al}$ interfaces but neglects the much enhanced in-plane magnetization of the CoFeB films due to increased thickness. Since the values of $n$ and $\mathrm{k}$ at $660 \mathrm{~nm}$ for $\mathrm{CoFeB}$ are almost the same as those for $\mathrm{W}$ (Figure 3), the thickness-dependent $\cos (\delta)$ spectrum of the $\mathrm{CoFeB} / \mathrm{AAO}$ thin films is very similar to that of the $\mathrm{W} / \mathrm{CoFeB} / \mathrm{W} / \mathrm{AAO}$ 
thin films. If the total thickness of the $\mathrm{W} / \mathrm{CoFeB} / \mathrm{W}$ layer (the addition of the CoFeB thickness and the total $\mathrm{W}$ layer thickness (the top layer and the bottom layer)) is used, the thickness-dependent $\cos (\delta)$ spectra match very well. As the ferromagnetic CoFeB layer is thin enough (e.g., $120 \mathrm{~nm}$ or less), the Kerr rotation may be dominated by the interference of the reflected light partially polarized by the surface magnetization of the CoFeB films, nearly following Equations (1)-(3), which may cause a complete destructive interference at a certain thickness and result in one null point for pure CoFeB thin films. With the further thickness increase of the CoFeB layer, the in-plane magnetization becomes increasingly strong. The in-plane magnetization will be the dominant factor to polarize the inducing light and increase the Kerr rotation angle. Even though the increased thickness still causes the interference of the reflected light, it will not be possible to reduce the L-MOKE rotation angle to zero due to the significantly enhanced polarization by the strong in-plane magnetization of the thick ferromagnetic CoFeB layer. As for W/CoFeB/W/AAO thin films, there will be no further increased in-plane magnetization besides the surface pining effect or possible spin-orbit coupling since $\mathrm{W}$ is a kind of non-ferromagnetic material, leading to an additional Kerr null point with the thickness increase of the W layer.
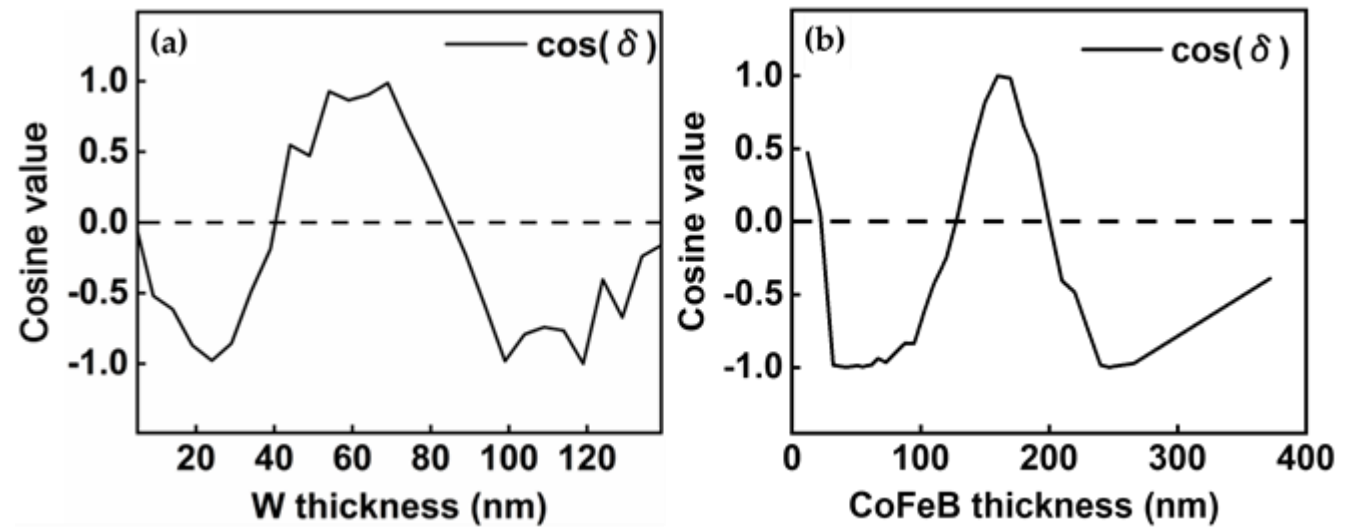

Figure 9. (a) The calculated $\cos (\delta)$ for the $\mathrm{W} / \mathrm{CoFeB}(120 \mathrm{~nm}) / \mathrm{W}$ thin films with the $\mathrm{W}$ layer thickness changing from 4 to $140 \mathrm{~nm}$; (b) the calculated $\cos (\delta)$ for the CoFeB/AAO thin films with a CoFeB layer thickness from 12 to $372 \mathrm{~nm}$.

As a result, introducing heavy metal layers of high spin-orbit interaction (e.g., W) on the top and/or bottom of the ferromagnetic layer provides a new type of system for obtaining Kerr rotation reversal, and the physical mechanism is different from that of manipulating the sign of Kerr rotation in magnetoplasmonic systems, in which the concerted action of nanoplasmonics and magnetization can control the sign of rotation of the reflected light's polarization in ferromagnetic nanostructures [8,55-57]. With respect to the interference pattern formed by light reflected from the air-CoFeB and $\mathrm{AAO} / \mathrm{Al}$ interfaces in the pure $\mathrm{CoFeB}(120 \mathrm{~nm}) / \mathrm{AAO} / \mathrm{Al}$ nanoporous thin film, this interference may be significantly affected by the enhanced in-plane magnetization of the film as the CoFeB thickness is increased more than a certain value. As the tungsten layers are introduced, no further enhanced magnetization can be introduced into the sandwich structural $\mathrm{W} / \mathrm{CoFeB} / \mathrm{W}$ thin film since the ferromagnetic CoFeB layer thickness is fixed but the pining effect (note: it will not be affected by the $W$ thickness increase too much) on the magnetization of CoFeB layer by the two $\mathrm{W}$ layers. The phase difference by the reflection coefficients $r_{p s}$ and $r_{S S}$ change due to the interference of the reflected light might lead to additional null points. In addition, a very thin layer of heavy metal can increase the L-MOKE rotation angle and the related magneto-optical susceptibility and reduce the coercivity. However, this study also confirms that improved inner magnetization and/or surface magnetization is still the main consideration for further enhanced Kerr rotation angle and magneto-optical susceptibility. 


\section{Conclusions and Perspectives}

Kerr rotation loop inversion (i.e., the Kerr null points) of nanoporous ferromagnetic $\mathrm{CoFeB} / \mathrm{AAO} / \mathrm{Al}$ multilayer systems with $\mathrm{CoFeB}$ layer thicknesses of 12 372 nm was revealed experimentally and theoretically. The $\mathrm{CoFeB}$ film showed a critical thickness of $51 \sim 55 \mathrm{~nm}$, at which a relative inversion of the Kerr hysteresis loops was observed. Furthermore, the introduction of W layers induced a second inversion of the Kerr signal in the system. The simulation of the phase difference between the reflection coefficient change caused by the interference of the reflected light and the theoretical analysis of the different inner magnetization dependent Kerr rotations for the pure $\mathrm{CoFeB} / \mathrm{AAO}$ thin films and $\mathrm{W} / \mathrm{CoFeB}(120 \mathrm{~nm}) / \mathrm{W} / \mathrm{AAO}$ thin films indicate that these Kerr null points are possibly determined by the combination effects of the in-plane magnetization and the interference of the reflected light. As for the $\mathrm{CoFeB} / \mathrm{AAO}$ systems, the interference of the reflected light will be dominated as the $\mathrm{CoFeB}$ layer is thin enough, but the in-plane magnetization will be increased greatly with the ferromagnetic $\mathrm{CoFeB}$ layer thickness increase, leading to only one Kerr null point. As for W/CoFeB $(120 \mathrm{~nm}) / \mathrm{W}$ nanoporous thin films, the phase difference between the reflection coefficients change caused by the interference of the reflected light is always dominated due to the non-ferromagnetic $\mathrm{W}$ layer. In addition, the introduction of $\mathrm{W}$ layers can greatly increase the near zero field magneto-optical susceptibility (the highest $\chi:-3.04 \mathrm{mdeg} / \mathrm{Oe} @ \mathrm{~W}=4 \mathrm{~nm}$ ) and the saturated Kerr rotation angle $\left(\theta_{\mathrm{S}}: 198.6 \mathrm{mdeg} @ \mathrm{~W}=74 \mathrm{~nm}\right)$ by construction of $\mathrm{W} / \mathrm{CoFeB}(120) / \mathrm{W}$ sandwich nanoporous thin films by comparing with the pure $\mathrm{CoFeB}$ thin films $(\chi=-1.21 \mathrm{mdeg} / \mathrm{Oe}$; $\left.\theta_{\mathrm{S}}=70 \mathrm{mdeg}\right)$. The coercivity of the $\mathrm{W} / \mathrm{CoFeB} / \mathrm{W}$ nanoporous thin film at a $\mathrm{W}$ thickness of $4 \mathrm{~nm}$ is $116.5 \mathrm{Oe}$, smaller than the pure CoFeB thin films with the same CoFeB thickness of $120 \mathrm{~nm}(130.5 \mathrm{Oe})$. Understanding these MO features of the multilayered ferromagnetic nanoporous thin film systems with non-magnetic metallic layers of various thicknesses for tunable $\mathrm{MO}$ effects and flexible phase modulation will pave a new approach for fabricating magnetic nanostructure devices for ultrasensitive magneto-optical sensing applications. Particularly, tunable MOKE effects and flexible phase modulation significantly rely on MOKE susceptibility, ellipticity, and reflectance (related to dielectric change or refractive index change), which are suitable for future ultrasensitive magnetometry, thickness sensors, and biosensors of high resolution.

Author Contributions: Data curation, formal analysis, funding acquisition, investigation, software, and writing-original draft preparation, W.Z.; formal analysis, investigation, resources, validation, and writing - review and editing, Z.C.; formal analysis, funding acquisition, investigation, methodology, project administration, resources, validation, writing-review and editing, V.I.B.; conceptualization, data curation, formal analysis, funding acquisition, investigation, methodology, project administration, resources, supervision, validation, writing - review and editing, Y.S. All authors have read and agreed to the published version of the manuscript.

Funding: This study was financially supported by BRICS STI Framework Programmed by NSFC (No. 51861145309) and RFBR (No.18-52-80038); by the Ministry of Science and Higher Education of the Russian Federation, Megagrant project N 075-15-2019-1934; the National Natural Science Foundation of China (No. 51971029); and National S \& T Major Project of China (No. 2018ZX10301201). This study is partially supported by the China Postdoctoral Science Foundation (No. 2020M680336) and the Postdoctor Research Foundation of Shunde Graduate School of the University of Science and Technology Beijing (No. 2020BH005).

Institutional Review Board Statement: Not applicable.

Informed Consent Statement: Not applicable.

Data Availability Statement: Data sharing is not applicable to this article.

Conflicts of Interest: There are no conflict of interest to declare among authors. 


\section{References}

1. Nikitov, S.A.; Kalyabin, D.V.; Lisenkov, I.V.; Slavin, A.N.; Pavlov, E.S. Magnonics: A new research area in spintronics and spin wave electronics. Phys.-Uspekhi 2015, 58, 1099-1128. [CrossRef]

2. Zimnyakova, P.E.; Ignatyeva, D.O.; Karki, D.; Voronov, A.A.; Shaposhnikov, A.N.; Berzhansky, V.N.; Levy, M.; Belotelov, V.I. Twodimensional array of iron-garnet nanocylinders supporting localized and lattice modes for the broadband boosted magneto-optics. Nanophotonics 2021, 11, 119-127. [CrossRef]

3. Gabbani, A.; Petrucci, G.; Pineider, F. Magneto-optical methods for magnetoplasmonics in noble metal nanostructures. J. Appl. Phys. 2021, 129, 211101. [CrossRef]

4. Rave, W.; Zueco, E.; Schäfer, R.; Hubert, A. Observations on high-anisotropy single crystals using a combined Kerr/magnetic force microscope. J. Magn. Magn. Mater. 1998, 177-181, 1474-1475. [CrossRef]

5. Wolfgang, R.; Rudolf, S.; Alex, H. Quantitative observation of magnetic domains with the magneto-optical Kerr effect. J. Magn. Magn. Mater. 1987, 65, 7-14. [CrossRef]

6. Hoffmann, V.; Schäfer, R.; Appel, E.; Hubert, A.; Soffel, H. First domain observations with the magneto-optical Kerr effect on Ti-ferrites in rocks and their synthetic equivalents. J. Magn. Magn. Mater. 1987, 71, 90-94. [CrossRef]

7. Buchanan, K.S.; Krichevsky, A.; Freeman, M.R.; Meldrum, A. Magnetization dynamics of interacting iron nanocrystals in $\mathrm{SiO}_{2}$. Phys. Rev. B 2004, 70, 174436. [CrossRef]

8. Sepúlveda, B.; Calle, A.; Lechuga, L.M.; Armelles, G. Highly sensitive detection of biomolecules with the magneto-optic surface-plasmon-resonance sensor. Opt. Lett. 2006, 31, 1085-1087. [CrossRef]

9. Rizal, C. Magneto-optic SPR-based Biosensors. In Proceedings of the 2020 Photonics North (PN), Niagara Falls, ON, Canada, 26-28 May 2020; p. 1. [CrossRef]

10. Rizal, C.; Belotelov, V. Sensitivity comparison of surface plasmon resonance (SPR) and magneto-optic SPR biosensors. Eur. Phys. J. Plus 2019, 134, 1-6. [CrossRef]

11. Ayareh, Z.; Mahmoodi, S.; Moradi, M. Magneto-plasmonic biosensing platform for detection of glucose concentration. Optik 2019, 178, 765-773. [CrossRef]

12. David, S.; Polonschii, C.; Luculescu, C.; Gheorghiu, M.; Gáspár, S.; Gheorghiu, E. Magneto-plasmonic biosensor with enhanced analytical response and stability. Biosens. Bioelectron. 2015, 63, 525-532. [CrossRef]

13. Chandra, S.; Cozart, J.; Biswas, A.; Lee, S.; Chanda, D. Magnetoplasmons for Ultrasensitive Label-Free Biosensing. ACS Photonics 2021, 8, 1316-1323. [CrossRef]

14. You, W.; Tengdin, P.; Chen, C.; Shi, X.; Zusin, D.; Zhang, Y.; Gentry, C.; Blonsky, A.; Keller, M.; Oppeneer, P.M.; et al. Revealing the Nature of the Ultrafast Magnetic Phase Transition in Ni by Correlating Extreme Ultraviolet Magneto-Optic and Photoemission Spectroscopies. Phys. Rev. Lett. 2018, 121, 077204. [CrossRef] [PubMed]

15. Belyaev, V.K.; Rodionova, V.V.; Grunin, A.A.; Inoue, M.; Fedyanin, A.A. Magnetic field sensor based on magnetoplasmonic crystal. Sci. Rep. 2020, 10, 7133. [CrossRef] [PubMed]

16. Campo, G.; Pineider, F.; Bonanni, V.; Albino, M.; Caneschi, A.; de Julián Fernández, C.; Innocenti, C.; Sangregorio, C. Magnetooptical probe for investigation of multiphase Fe oxide nanosystems. Chem. Mater. 2015, 27, 466-473. [CrossRef]

17. Baselt, D.R.; Lee, G.U.; Natesan, M.; Metzger, S.W.; Sheehan, P.E.; Colton, R.J. A biosensor based on magnetoresistance technology. Biosens. Bioelectron. 1998, 13, 731-739. [CrossRef]

18. Buznikov, N.A.; Safronov, A.P.; Orue, I.; Golubeva, E.V.; Lepalovskij, V.N.; Svalov, A.V.; Chlenova, A.A.; Kurlyandskaya, G.V. Modelling of magnetoimpedance response of thin film sensitive element in the presence of ferrogel: Next step toward development of biosensor for in-tissue embedded magnetic nanoparticles detection. Biosens. Bioelectron. 2018, 117, 366-372. [CrossRef]

19. Brzeska, M.; Panhorst, M.; Kamp, P.B.; Schotter, J.; Reiss, G.; Pühler, A.; Becker, A.; Brückl, H. Detection and manipulation of biomolecules by magnetic carriers. J. Biotechnol. 2004, 112, 25-33. [CrossRef]

20. Chen, L.; Bao, C.-C.; Yang, H.; Li, D.; Lei, C.; Wang, T.; Hu, H.-Y.; He, M.; Zhou, Y.; Cui, D.-X. A prototype of giant magnetoimpedance-based biosensing system for targeted detection of gastric cancer cells. Biosens. Bioelectron. 2011, 26, 3246-3253. [CrossRef]

21. Yang, Z.; Wang, H.; Dong, X.; Yan, H.; Lei, C.; Luo, Y. Giant magnetoimpedance based immunoassay for cardiac biomarker myoglobin. Anal. Methods 2017, 9, 3636-3642. [CrossRef]

22. Caballero, B.; García-Martín, A.; Cuevas, J.C. Hybrid magnetoplasmonic crystals boost the performance of nanohole arrays as plasmonic sensors. ACS Photonics 2016, 3, 203-208. [CrossRef]

23. Manera, M.G.; Ferreiro-Vila, E.; Garcia-Martin, J.M.; Garcia-Martin, A.; Rella, R. Enhanced antibody recognition with a magnetooptic surface plasmon resonance (MO-SPR) sensor. Biosens. Bioelectron. 2014, 58, 114-120. [CrossRef]

24. Schmitte, T.; Theis-Br\$ouml\$hl, K.; Leiner, V.; Zabel, H.; Kirsch, S.; Carl, A. Magneto-optical study of the magnetization reversal process of Fe nanowires. J. Phys. Condens. Matter 2002, 14, 7525-7538. [CrossRef]

25. Tillmanns, A.; Oertker, S.; Beschoten, B.; Guentherodt, G.; Leighton, C.; Schuller, I.K.; Nogues, J. Magneto-optical study of magnetization reversal asymmetry in exchange bias. Appl. Phys. Lett. 2006, 89, 202512. [CrossRef]

26. Huang, D.; Lattery, D.; Wang, X. Materials engineering enabled by time-resolved magneto-optical kerr effect for spintronic applications. ACS Appl. Electron. Mater. 2021, 3, 119-127. [CrossRef] 
27. Zeng, H.T.; Read, D.; Petit, D.; Jausovec, A.V.; O’Brien, L.; Lewis, E.R.; Cowburn, R.P. Combined electrical and magneto-optical measurements of the magnetization reversal process at a domain wall trap. Appl. Phys. Lett. 2009, 94, 103113. [CrossRef]

28. Sedrpooshan, M.; Ahmadvand, H.; González, D.L.; van Dijken, S. Magneto-optical study of anomalous magnetization reversal in the presence of anisotropy dispersion in CoPd thin films. Phys. Rev. B 2018, 98, 214444. [CrossRef]

29. Beaurepaire, E.; Merle, J.C.; Daunois, A.; Bigot, J.Y. Ultrafast spin dynamics in ferromagnetic nickel. Phys. Rev. Lett. 1996, 76, 4250-4253. [CrossRef]

30. Lambert, C.-H.; Mangin, S.; Varaprasad, B.S.D.C.S.; Takahashi, Y.K.; Hehn, M.; Cinchetti, M.; Malinowski, G.; Hono, K.; Fainman Y.; Aeschlimann, M.; et al. All-optical control of ferromagnetic thin films and nanostructures. Science 2014, 345, 1337-1340. [CrossRef]

31. Zdorovets, M.V.; Kozlovskiy, A.L.; Shlimas, D.I.; Borgekov, D.B. Phase transformations in $\mathrm{FeCo}_{-}-\mathrm{Fe}_{2} \mathrm{CoO}_{4} / \mathrm{Co} 3 \mathrm{O}_{4}$-spinel nanostructures as a result of thermal annealing and their practical application. J. Mater. Sci. Mater. Electron. 2021, 32, 16694-16705. [CrossRef]

32. Vinnik, D.A.; Starikov, A.Y.; Zhivulin, V.E.; Astapovich, K.A.; Turchenko, V.A.; Zubar, T.y.I.; Trukhanov, S.V.; Kohout, J.; Kmječ, T.; Yakovenko, $\mathrm{O}$.; et al. Changes in the structure, magnetization, and resistivity of $\mathrm{BaFe}_{12-\mathrm{x}} \mathrm{Ti}_{\mathrm{x}} \mathrm{O}_{19}$. ACS Appl. Electron. Mater. 2021, 3, 1583-1593. [CrossRef]

33. Feldmann, J.; Youngblood, N.; Wright, C.D.; Bhaskaran, H.; Pernice, W.H.P. All-optical spiking neurosynaptic networks with self-learning capabilities. Nature 2019, 569, 208-214. [CrossRef] [PubMed]

34. Liu, X.D.; Xu, Z.; Gao, R.X.; Chen, Z.F.; Lai, T.S.; Du, J.; Zhou, S.M. Improved time-resolved magneto-optical Kerr effect technique and dynamic magnetization reversal mechanism of perpendicularly magnetized L10 FePt films. arXiv 2009, arXiv:0812.1360.

35. Lei, C.X.; Man, Z.S.; Tang, S.L. Extraordinary optical transmission and enhanced magneto-optical faraday effect in the cascaded double-fishnet structure with periodic rectangular apertures. Curr. Opt. Photonics 2020, 4, 134-140. [CrossRef]

36. Li, N.; Tang, T.; Li, J.; Luo, L.; Sun, P.; Yao, J. Highly sensitive sensors of fluid detection based on magneto-optical optical Tamm state. Sens. Actuators B Chem. 2018, 265, 644-651. [CrossRef]

37. Borovkova, V.; Ignatyeva, D.O.; Sekatskii, S.K.; Karabchevsky, A.; Belotelov, V.I. High-Q surface electromagnetic wave resonance excitation in magneto-photonic crystals for super-sensitive detection of weak light absorption in near-IR. Photonics Res. 2020, 8 , 57-63. [CrossRef]

38. Qin, J.; Deng, L.; Xie, J.; Tang, T.; Bi, L. Highly sensitive sensors based on magneto-optical surface plasmon resonance in Ag/CeYIG heterostructures. AIP Adv. 2015, 5, 017118. [CrossRef]

39. Knyazev, G.A.; Kapralov, P.O.; Gusev, N.A.; Kalish, A.N.; Belotelov, V.I. Magnetoplasmonic Crystals for Highly Sensitive Magnetometry. ACS Photonics 2018, 5, 4951-4959. [CrossRef]

40. Tishkevich, D.I.; Grabchikov, S.S.; Lastovskii, S.B.; Trukhanov, S.V.; Zubar, T.I.; Vasin, D.S.; Trukhanov, A.V. Correlation of the synthesis conditions and microstructure for Bi-based electron shields production. J. Alloys Compd. 2018, 749, 1036-1042. [CrossRef]

41. Zubar, T.I.; Fedosyuk, V.M.; Trukhanov, S.V.; Tishkevich, D.I.; Michels, D.; Lyakhov, D.; Trukhanov, A.V. Method of surface energy investigation by lateral AFM: Application to control growth mechanism of nanostructured NiFe films. Sci. Rep. 2020, $10,14411$. [CrossRef]

42. Lordan, D.; Wei, G.; McCloskey, P.; O'Mathuna, C.; Masood, A. Origin of perpendicular magnetic anisotropy in amorphous thin films. Sci. Rep. 2021, 11, 3734. [CrossRef]

43. Zhang, W.; Wang, Y.; Wang, Q.; Belotelov, V.I.; Song, Y. Surface and Interface Engineering Multilayered Nanopore Films for Enhanced Fabry-Pérot Interferences. J. Phys. Chem. C 2018, 122, 29457-29463. [CrossRef]

44. Zhang, W.; Li, J.; Ding, X.; Pernod, P.; Tiercelin, N.; Song, Y. Tunable Magneto-Optical Kerr Effects of Nanoporous Thin Films. Sci. Rep. 2017, 7, 2888. [CrossRef] [PubMed]

45. Zhang, W.; Wang, Q.; Zhao, C.; Song, Y. The optical cavity enhanced magneto-optical Kerr effect signals of AAO/Al-based CoFeB nanostructure arrays. Opt. Commun. 2019, 437, 44-49. [CrossRef]

46. Hoffmann, M.A.; Sharma, A.; Matthes, P.; Okano, S.; Hellwig, O.; Ecke, R.; Zahn, D.R.T.; Salvan, G.; Schulz, S.E. Spectroscopic ellipsometry and magneto-optical Kerr effect spectroscopy study of thermally treated $\mathrm{Co}_{60} \mathrm{Fe}_{20} \mathrm{~B}_{20}$ thin films. J. Phys. Condens. Matter 2020, 32, 055702. [CrossRef]

47. Sharma, A.; Hoffmann, M.A.; Matthes, P.; Hellwig, O.; Kowol, C.; Schulz, S.E.; Zahn, D.R.T.; Salvan, G. Crystallization of optically thick films of $\mathrm{Co}_{x} \mathrm{Fe}_{80-x} \mathrm{~B}_{20}$ : Evolution of optical, magneto-optical, and structural properties. Phys. Rev. B 2020, 101, 776. [CrossRef]

48. Song, Y.; Wang, Y.; Li, B.B.; Fernandes, C.; Ruda, H.E. Interface interaction induced ultra-dense nanoparticles assemblies. Nanoscale 2013, 5, 6779-6789. [CrossRef] [PubMed]

49. Hindmarch, A.T.; Rushforth, A.W.; Campion, R.P.; Marrows, C.H.; Gallagher, B.L. Origin of in-plane uniaxial magnetic anisotropy in CoFeB amorphous ferromagnetic thin-films. Phys. Rev. B 2011, 83, 1417-1425. [CrossRef]

50. García, D.; Muñoz, J.L.; Kurlyandskaya, G.; Vázquez, M.; Ali, M.; Gibbs, M.R.J. Induced anisotropy, magnetic domain structure and magnetoimpedance effect in CoFeB amorphous thin films. J. Magn. Magn. Mater. 1999, 191, 339-344. [CrossRef]

51. Diaz-Valencia, B.F.; Mejia-Salazar, J.R.; Oliveira, O.N., Jr.; Porras-Montenegro, N.; Albella, P. Enhanced Transverse MagnetoOptical Kerr Effect in Magnetoplasmonic Crystals for the Design of Highly Sensitive Plasmonic (Bio)sensing Platforms. ACS Omega 2017, 2, 7682-7685. [CrossRef] [PubMed]

52. Zak, J.; Moog, E.R.; Liu, C.; Bader, S.D. Universal approach to magneto-optics. J. Magn. Magn. Mater. 1990, 89, 107-123. [CrossRef] 
53. Rakić, A.D. Algorithm for the determination of intrinsic optical constants of metal films: Application to aluminum. Appl. Opt. 1995, 34, 4755-4767. [CrossRef] [PubMed]

54. Zhang, W.; Song, Y. Fundamental in Thin Films and Coatings, 1st ed.; Song, Y., Ed.; Wiley-VCH: Weinheim, Germany, 2021; Volume 1, pp. 66-75. [CrossRef]

55. Sepulveda, B.; Gonzalez-Diaz, J.B.; Garcia-Martin, A.; Lechuga, L.M.; Armelles, G. Plasmon-induced magneto-optical activity in nanosized gold disks. Phys. Rev. Lett. 2010, 104, 147401. [CrossRef]

56. Bonanni, V.; Bonetti, S.; Pakizeh, T.; Pirzadeh, Z.; Chen, J.; Nogues, J.; Vavassori, P.; Hillenbrand, R.; Akerman, J.; Dmitriev, A. Designer magnetoplasmonics with nickel nanoferromagnets. Nano Lett. 2011, 11, 5333-5338. [CrossRef] [PubMed]

57. Chen, J.; Albella, P.; Pirzadeh, Z.; Alonso-Gonzalez, P.; Huth, F.; Bonetti, S.; Bonanni, V.; Akerman, J.; Nogues, J.; Vavassori, P.; et al. Plasmonic nickel nanoantennas. Small 2011, 7, 2341-2347. [CrossRef] 